\title{
Un sermón en quechua de Diego de Molina (Huánuco, 1649)
}

Un sermon en quechua de Diego de Molina (Huánuco, 1649)

A sermon in Quechua by Diego de Molina (Huánuco, 1649)

\section{Gerald Taylor}

\section{QpenEdition}

\section{Journals}

\section{Edición electrónica}

URL: http://journals.openedition.org/bifea/7032

DOI: $10.4000 /$ bifea.7032

ISSN: 2076-5827

Editor

Institut Français d'Études Andines

Edición impresa

Fecha de publicación: 1 agosto 2001

Paginación: 211-231

ISSN: 0303-7495

\section{Referencia electrónica}

Gerald Taylor, « Un sermón en quechua de Diego de Molina (Huánuco, 1649) », Bulletin de l'Institut français d'études andines [En línea], 30 (2) | 2001, Publicado el 08 agosto 2001, consultado el 08

diciembre 2020. URL : http://journals.openedition.org/bifea/7032 ; DOI : https://doi.org/10.4000/bifea 7032

\section{(c) (†) $\ominus$}

Les contenus du Bulletin de l'Institut français d'études andines sont mis à disposition selon les termes de la licence Creative Commons Attribution - Pas d'Utilisation Commerciale - Pas de Modification 4.0 International. 


\title{
UN SERMÓN EN QUECHUA DE DIEGO DE MOLINA (HUÁNUCO, 1649)
}

Gerald TAYLOR *

\section{Resumen}

Diego de Molina, cura de Huánuco, terminó la redacción de sus Sermones de la Cuaresma en Lengua quechua en febrero del año de 1649. Hasta ahora sólo se ha publicado el texto preliminar (Romero, 1928). Aquí presentamos la versión paleográfica del texto castellano original de un fragmento de uno de los sermones y su traducción contemporánea en quechua, tarea complicada por el hecho de que el manuscrito fue parcialmente deteriorado por el incendio de la Biblioteca Nacional del Perú en 1943. Presentamos igualmente una versión normalizada del texto quechua, acompañada de su traducción al castellano.

Palabras claves: Quechua, lingüística, paleografía, evangelización.

\section{UN SERMON EN QUECHUA DE DIEGO DE MOLINA (HUÁNUCO, 1649)}

\section{Résumé}

Diego de Molina, curé de Huánuco, termina de rédiger ses Sermons du carême en langue quechua en février 1649. Jusqu'à présent on n'en a publié que le texte préliminaire (Romero, 1928). Nous présentons ici la version paléographique du texte espagnol original d'un fragment de l'un des sermons et de sa traduction contemporaine en quechua, tâche compliquée du fait que le manuscrit a été partiellement détérioré par l'incendie de la Bibliothèque Nationale du Pérou en 1943. Nous présentons également une version normalisée du texte quechua accompagnée de sa traduction en espagnol.

Mots clés : Quechua, linguistique, paléographie, évangélisation.

\section{A SERMON IN QUECHUA BY DIEGO DE MOLINA (HUÁNUCO, 1649)}

\begin{abstract}
Diego de Molina, parish priest of Huánuco, finished writing his Sermons for Lent in Quechua in February, 1649. Up till now, only the preliminary text has been published (Romero, 1928). In this article, we present the paleographical version of the original Spanish text of a fragment of one of the sermons and its contemporary translation to Quechua, a task which has been complicated by the damage caused to the manuscript during the fire which destroyed the National Library of Peru in 1943. We also present a normalized version of the Quechua text accompanied by its Spanish translation.
\end{abstract}

Key words: Quechua, linguistics, paleography, evangelisation.

* IFEA: Casilla 18-1217, Lima 18 - Perú y Centre d'Études sur les Langues Indigènes d'Amérique (CELIA-CNRS), 7 rue Guy Moquet, B. P. 8, 94801 Villejuif, cedex, Francia. 
Los estudiosos de la filología quechua saben de la existencia del sermonario de Diego de Molina, manuscrito conservado en la Biblioteca Nacional de Lima, desde la publicación de "los preliminares de la obra" en la Revista Histórica en 1928 (Romero, 1928). La paleografía de Carlos A. Romero no corresponde a los criterios actuales, es inconsistente, faltan palabras y hay errores en la transcripción. Sin embargo, nos permite al menos tener una idea de cómo era una parte de este manuscrito de "327 hojas sin foliar, más 3 páginas en blanco" antes del deterioro parcial provocado por el incendio de la Biblioteca y el daño causado por el agua utilizada para apagarlo. Como se puede apreciar en la fotocopia que acompaña el sermón que presentamos aquí, aun cuando una de las esquinas es ilegible, el resto del texto resulta lo suficientemente claro como para poder ser interpretado sin mucho esfuerzo. No es el caso de todo el manuscrito y, sobre todo, de la parte preliminar transcrita por Romero que contiene, hoy, muchas lagunas.

El manuscrito de Molina es una de las tres grandes colecciones de sermones redactadas en quechua al final de los años cuarenta del siglo XVII. Las otras son el sermonario de Francisco de Ávila y el de Fernando de Avendaño. Ambos fueron publicados en esa época. No sabemos por qué el sermonario de Diego de Molina no tuvo la misma suerte. Tal vez la influencia de los franciscanos no pudo competir con la de los jesuitas en aquel entonces. Los sermones correspondían a las fiestas de la Cuaresma. El extracto que publicamos aquí pertenece al Sermón del Domingo de Pascua de Resurrección y describe la bajada del Espíritu Santo sobre los apóstoles en Jerusalén y predice cómo será el Juicio Universal. Hemos escogido este pasaje porque se trata de uno de los fragmentos más fácilmente reconstituibles del texto y porque se concluye con el comentario del autor sobre la lengua, previamente transcrito por Romero y publicado en el artículo ya citado (Romero, 1928: 53).

¿Quién era Diego de Molina? En realidad, pocos son los datos que se refieren al autor del sermonario. Como lo menciona Romero, la "crónica franciscana, escrita en 1638, por el cronista del orden, Fr. Diego de Córdoba ... se contiene una relación de escritores de la Orden, en la cual no figura aún el nombre del P. Molina, ni tan poco en la de los sucesos de los misioneros en Huánuco" (Romero, 1928: 51). Investigaciones — superficiales - en archivos tampoco nos han ayudado. Según el manuscrito, Diego de Molina era cura en Huánuco en los postrimeros años 40 del siglo XVII. El historiador Ramón Mujica nos ha señalado una entrada en el Diccionario Histórico-Cronológico... de Luis Antonio Eguiguren (1949) que menciona entre los alumnos que ingresaron al colegio de San Martín en el año de 1609:

"Diego de Molina, de Guamanga. Entró en 10 de Enero de 12 años. Fue religioso de San Francisco".

Sigue

"Luis de Molina. Fue hermano del antecedente y entró en el mismo día de 11 años" (Eguiguren, 1949: 149).

Significa que, si se trata del mismo Diego de Molina, lo que es muy probable, cuando terminó la redacción de su sermonario en 1649, ya había cumplido 52 años. Otra referencia en el mismo libro indica que

"Los escribanos de Su Majestad que, aquí firmamos, certificamos que el licenciado Diego de Molina, de quien vá firmado y autorizado este 
testimonio, es tal escribano público como se nombra, y que a sus autos y testimonios que van autorizados y firmados de su nombre, se les dá entera fé y crédito..." (Eguiguren, 1949: 769).

Este testimonio es del año 1625.

El comentario preliminar al sermonario, bien conocido y frecuentemente citado gracias al artículo de Romero, ha inducido a algunos autores a la falsa suposición de considerar que Molina refleja las preocupaciones lingüísticas de su época. Sin embargo, no obstante las críticas que hace de la transcripción ambigua del Salve Regina, del Credo y del Ave María en las que no se representa "la gutural" (/q/), él mismo tampoco la indica en sus sermones ni por el empleo tradicional de $c c$ y $q q u$ ni por la grafía que aparentemente le parece más apropiada, la colocación de un circunflejo sobre $c$ y $q$. Esta última variante aparece en otras obras de la misma época. En el léxico que proporciona de las palabras que se distinguen por el contraste entre $/ \mathrm{k} / \mathrm{y} / \mathrm{q} /$, indica este último fonema no por el circunflejo sino por la grafía tradicional de $c c$ y $q q u$. Transcribe el fonema /ch/ por la grafía çh. Así, evita que se confunda con $c$ en final de sílaba seguida por el apoyo gráfico $h$, como en mamançhichuan (/mamanchik-wan/). Aunque el estilo de la introducción y el de los sermones reflejen ambos lo florido del barroco, hay una diferencia notable entre los dos. La presentación es relativamente confusa y las normas gráficas propuestas poco coherentes (como en el caso de la representación de las "guturales"). Los sermones, al contrario, redactados según la tradición más ortodoxa de la lengua general del III Concilio, siguen un modelo clásico. Aquí, el "barroco" corresponde al siglo precedente y poco lo distingue del estilo que Oré emplea en su Symbolo Catholico Indiano.

Nuestro colega, Xavier Pello, en una comunicación personal, ha sugerido la probable influencia del sermonario perdido de Oré sobre la redacción del manuscrito de Molina. Efectivamente, Molina transcribe un rezo de Oré debajo del cual pone su propio nombre. En aquella época, eso no significaba necesariamente un plagio y el mismo Molina escribe:

"Bien se acuerda el Sr. de premiar al illustríssimo señor P. Fr. Luis Hierónimo de Oré, obispo digníssimo que tanto trabajó por el bien de las almas, y nos dejó tan lucidas obras." (Romero, 1928: 61).

Es comprensible que el máximo escritor franciscano de la evangelización andina haya inspirado a un redactor de sermones perteneciente a la misma orden. Tal vez, por eso, el estilo y el lenguaje de los sermones contrasten con las normas propuestas en la introducción. Lo que es cierto es que la ortografía de los sermones corresponde totalmente a las reglas establecidas por los lingüistas del III Concilio Limense y que no hay influencia alguna de un dialecto "chinchaysuyo", tal como se habría debido hablar en Huánuco en aquella época. Dice Molina en el f. 302v:

"La traducción de los Santos Evangelios según la letra y el sentido va regulada en todos los términos con el idioma latino, y correcta según el parecer de las personas más hábiles que he podido hallar en la lengua, con los cuales se ha tratado y comunicado y dificultado muchas veces y la han aprobado sacerdotes clérigos y religiosos los mejores lenguas que 
he podido comunicar, A seglares montañeses e indios muy ladinos ha parecido muy propia la traducción, así en Lima, Huamanga, Huánuco y Jauja y en otras partes. En todo he procurado siempre seguir la letra y el sentido y cuando no fuere posible seguir la letra, jamás habrá falta en seguir el sentido." (Grafía modernizada, véase la versión paleográfica que presentamos en este artículo).

La lengua a la que Molina se refiere, conocida entonces en todas las provincias del antiguo Chinchaysuyo citadas en su texto, era la "general", aparentemente aún comprendida en gran parte del territorio andino.

Presentamos aquí la transcripción paleográfica de un fragmento de un sermón del manuscrito de Molina. En el f, 298r empieza el texto castellano, modelo del sermón quechua; éste empieza en el f. 300r y acaba en el 302v. Sigue el comentario de Molina sobre la naturaleza del idioma empleado y su sumisión total a la voluntad de la iglesia, Finalmente, en el f. 303v, se transcribe en quechua una "oración en reverencia de la Santa Sábana, en que fue envuelto el cuerpo santísimo de Nuestro Señor Jesucristo". Concluimos nuestro estudio con una versión normalizada de ambos textos en quechua (el sermón y la oración) con traducción castellana enfrente.

En el texto paleográfico empleamos los símbolos siguientes:

/ = final de renglón y // final de página del manuscrito original;

[[ ]] indica un pasaje ilegible que ha sufrido los efectos del incendio. Cuando encierra un texto en cursivas, éste ha podido reconstruirse gracias a la versión correspondiente en una de las dos lenguas en que fue redactado. En el resto del documento, las letras en cursivas indican formas probables y las que están en negritas y cursivas son citas sin modificaciones de la paleografía de Romero;

< > representa una modificación del texto realizada por el autor del manuscrito;

[ ] es una adición al texto hecha por el autor del artículo; corresponde a una hipótesis justificada por el contexto.

Hemos trabajado principalmente sobre la base de las fotocopias. Sin embargo, para leer las partes ennegrecidas por el fuego, hemos tenido que consultar el microfilm. Por eso, la fotocopia que publicamos aquí sólo da una idea parcial del texto.

Hay muchos comentarios que se podría hacer sobre el contenido del manuscrito. Los reservamos para otro trabajo. Hemos estudiado este manuscrito desde 1982 y nos parecía que no deberíamos tardar más en publicar al menos un fragmento de nuestro trabajo. Esperamos todavía poder terminar una versión lo más completa posible del manuscrito en un futuro no demasiado distante. Otro extracto será publicado en una antología de textos de la evangelización en lengua general La lucha contra los huacas en preparación. 


\section{Extracto del Sermon del Domingo de Pascua de Resurreccion de P. Fr. Diego de Molina, 1649*}

(f298r) [[...]] a los quarenta dias en presencia de su sanc-/ (1) [[tissim]]a madre y destos sagrados Apostoles se subio a los cielos / [y s]e asento a la diestra de Dios. $\mathrm{P}(a d r)$ e poderoso. Al tiempo que iba / subiendo a los cielos bajaron al camino y le salieron a rreçebir / innumerable multitud de Angeles que baja-/ron, fueron muçhos mas que dies millares de millares ale-/grandose con grande rregosijo.

Con este acompañamiento subio jesuxp (ist)o s(eñor) n(uestro) a la gloria y (2) / bolo mas q(ue) los Seraphines.y. bolando mas q(ue) las plumas de los / vientos subio a los cielos: fue delante del rrebaño de su ganado / descubriendoles el camino, abrio las puertas de la gloria para $\mathrm{q}(u e) /$ entrasen los suyos.

Adoramosle Señor jesuxp(ist)o. q(ue) subiste a los cielos, y tambien te / adoramos asentado a la diestra de Dios $\mathrm{P}($ adre $)$. No olvides a mi peca-/dor pues eres Dios mio, mi salud, y mi Pastor; no dexes. ni des-/ampares a mi que soy la obeja perdida, llamame. y llebame / a ti. Con todo coraçon creo en Dios $\mathrm{P}(a d r)$ e y tanbien creo en su / vnico hijo, mi señor jesuxp(ist)o, y asimesmo creo en el $\operatorname{sp}($ irit $) \mathrm{u}$ s(an)to / que para siempre es eterno Dios.

El qual $\mathrm{sp}($ irit $) \mathrm{u} \mathrm{S}($ an)to poderoso. y Riquissimo Dios n(uest)ro. el dia de / Pentecostes (quando fueron cumplidos ya sinquenta dias ) (3) bajo / a los Apostoles; los quales despues de la Ascension de jesu (4) / xp(ist)o s(eñor) n(uestro) se auian vuelto a jerusalen a esperar en aquel lugar / la venida del spiritu sancto. Perseberaban juntos y conformes / en ayunos. y oraçiones dentro del Çenaculo juntamente con la / virgen Maria n(uest)ra vnica señora y Madre.

Como estuuiessen todos en aquel lugar rrepentinam(en)te vino el / spiritu sancto, y apareçio sobre cada vno dellos en lenguas de fuego(5). / Aquella casa donde se auian juntado quedo llena con la diuina / presençia del spiritu sancto, y sus almas fueron llenas de graçia / y consolaçion diuina. Oyendo aquesta voz y sonido todos los que (6) / estaban en jerusalen se juntaron, pensando. que podria ser? / y oyeron todos predicar a los Apostoles./

\section{$<$ Auian-> //}

(f298v) Auian se juntado de todas partes y de todas las [[... ] / y Naçiones muçhos hombres para adorar a Dios en jerusal[[en]] / el dia de Pascua de Pentecostes. Como predicasen los Apo[[s]]/toles, todos los hombres $\mathrm{q}(u e)$ alli estaban de diuersas naçiones / oyeron su predicaçion en la propria lengua de cada vno de los $\mathrm{q}(u e)$ / oyan.

(*) Se trata del último sermón de los Sermones de la quaresma en lengua quechua por el p. FR. Diego de Molina, de la orden de san francisco, cura de guanuco, etc. Año de 1649. Manuscrito B203 de la BNP.

(1) N.M. Actos 1.

(2) N.M. Psal. 17 / Mich. 2

(3) Signo casi ilegible; tal vez cierre el paréntesis.

(4) N.M. Act. 2.

(5) N.M. Act. 4.

(6) N.M. Act. 2. 
Porq(ue) segun lo que el Spiritu Sancto les enseñaba / hablaban los Apostoles en varias diuersas lenguas. / oyendolos muçhos hombres de los q(ue) alli estaban se conuirtie-/ron a Dios por su Doctrina.

Spiritu S(anc)to Dios llama ençendida y lumbre de Dios, enuia / y derrama esa llama y lumbre dentro de mis huessos y enseñame pa-/ra que me ençienda. e inflame en tu amor y graçia. Spiritu $\mathrm{S}(a n c)$ to / $\mathrm{P}(a d r)$ e de los pobres, ruego te dignes; y quieras. venir a mi pobre tuyo / y ablandes mi coraçon empedernido. y me hagas cessar de ofen/ derte. Spiritu sancto Dios rruegote quieras llouer el rrosio / (7) de tu diuina graçia. sobre los christianos heredad tuya.

Spiritu s(anc)to Dios, $\mathrm{q}(u e)$ con el $\mathrm{P}(a d r)$ e y con el hijo eres vn solo Dios, / perdona mis pecados pues tu eres verdadero perdonador de ellos / Spiritu sancto, aliuio, y ayuda de los cansados. ayudame, pues / estoy cansado con la carga de mis pecados: muçho me e cansado:/ señor caminando por el camino de mis pecados. Spiritu S(anc)to / fuente de aguas viuas, laba mi coraçon suçio. y harta mi ani-/ma: se sienta con tu diuina graçia. Spiritu sancto, suplicote: / los sembrados de mi alma, y las plantas marçhitas y secas, las / quieras rregar, pues con tu rriego rreuerdeçeran . y como los ver-/geles produçiran; y floreçeran.

Quiero acabar hijos mios este sermon con aduertiros; que / a de venir N(uestro) $\mathrm{s}($ eñor $)$ jesu $\mathrm{xp}($ ist $) \mathrm{o}$ al fin del mundo a jusgar a / todo el genero humano; entonçes rresucitaran todos los / muertos; y vendran a juizio. y a cada vno dara dios el pre-/mio conforme sus obras.//

(f299r) [[...] ] hijos mios y todos emos de rresuçitar çertissimam(en)te / [[...]] $\mathrm{q}(u e)$ dios señalare. con estos mismos cuerpos y con estos ojos, / [[y]] con estas manos, y con estos huessos, y con esta carne y con este / pellexo: No se perdera ni se trocara vn cabello por virtud / de aquel gran Dios. Quando ayan resuçitado todos los muertos. / y salido de los monumentos a la voz del hijo de Dios, entonces / enuiara Dios sus Angeles para $\mathrm{q}(u e)$ los junten en vn lugar a / todos los hombres i estando alli todos desde el primero hasta el vltimo de los mortales aguardando el juizio postrero, / apareçera en los cielos la señal de la sancta Cruz. y tras / ella vernan millares de Angeles. y sanctos con sus Espadas / afiladas en sus manos, y en medio dellos el hijo de dios jesu/xp(ist)o, juez vniuersal, verna con tanta magestad que los mismos / cielos temblaran de el.

Viendo los hombres al hijo de Dios abajaran sus ojos co-/mo muertos de espanto y diran: O montes cubridnos, Abysmos / sepultadnos: porque no veamos la yra de Dios. Asentado jesu-/xp(ist)o s(eñor) n(uestro) en su glorioso trono rrodeado de los exercitos çelestiales: / mandara a sus Angeles apartar los buenos de los malos, y al / punto los buenos seran puestos a la mano dereçha, y los malos / a la siniestra: O q(ue) sentiran los malos quando se vean eçhar / y arroxar al lado de la condenaçion . O que tristesa y quebranto / cubrira sus coraçones, como temblaran, y cruxiran los di/entes aunq(ue) ayan sido incas, y Emperadores.

Alli se sacaran hijos mios los libros en q(ue) estan escritos / las obras buenas y malas de cada vno; y por obra admirable / cada vno leera alli su vida, y leera todas las

(7) N.M. Psalm. / 61. (?) 
vidas de los otros / y vera quien mereçe muerte eterna y quien vida eterna. que / sentiran los malos quando vean voluerse a ellos el juez eter-/no con rostro ayrado. y con voz terrible decirles. Id malditos / enemigos mios al ynfierno para ser atormentados con / fuego eterno para siempre sin fin.

El hijo de Dios voluiendo despues sus amorosos ojos y su / rrostro muy alegre a sus escogidos: les dira dulcemente. Venid /

$<\operatorname{con}>/ /$

(f299v) conmigo benditos y qu[[eridos de mi padre ...]] / çielos que os tengo aparejado. $\mathrm{O} q(u e)$ goço inefable entrara en a/quellos coraçones, yran triumphando, y todos llenos de amor / y de contento viendo acabados ya sus trauajos; que ya no ay / hambre, ni enfermedad, ni pobreça, ni cansançio, ni muerte / ya no ay tristesa, ni cuydado. ni rrezelos. ya no ay pecados, / ni sensaçiones, ni mentiras, ni escandalos.

Todo esto se acaba, paso el Tiempo, y sus mudanças. Todo / es ya eternidad y paz, amor, goço, y descanso en aquellas moradas. / eternas, en aquellos prados floridos, en aquellas fuentes de / aguas viuas, en aquel rrio de deleytes que mana de Dios. y / donde beben sin çessar, siempre gustan mas. y siempre / beben deleytes de Dios. Sus Cuerpos seran mas ligeros que / las aguilas, mas rresplandeçientes que el sol, mas subtiles / que el viento. mas hermosos q(ue) el çielo, sus animas seran / como Dios, llenas del mismo Dios. yguales a los Angeles, hijos / queridos y rregalados de su Dios.

Todos tendran entre si entranable amor: dando cada vno a / los otros todo el bien q(ue) tiene, goçandose todos del bien de ca-/da vno, y cada vno goçando los bienes de todos: I sobre todo vi-/endo, y goçando los Thesoros de toda la hermosura, y suauidad / de nuestro Dios. No se puede hermanos mios pensar a esto, / como es, y muçho menos puede deçirles. Porq (ue) ni oyo oydo, ni / oyo. vio, ni ymagino pensamiento la grandesa de los bienes / que Dios tiene para los q(ue) le aman y siruen.

Amad hijos mios muçho a N(uestro) s(eñor) jesuxp(ist)o. seruilde con / todas vuestras fuerças, cumplid sus mandamientos aunque / os cueste la vida. esto q(ue) os e diçho hijos mios es nuestra fee / y esto es lo q $(u e)$ creemos, lo qual si alguno no creyere no podra / alcançar la salud de su anima, y saluaçion en Dios para siem/pre. Bien abenturados, si asi lo haçeis, sereis de los queridos / hijos de dios, gozareis aquella vida eterna $\mathrm{q}(u e)$ a los $\mathrm{q}(u e)$ le sir-/uen fielmente promete $\mathrm{n}($ uest $)$ ro señor Jesuchristo. //

(f300r) [[el]] qual con el $\mathrm{P}(a d r)$ e y con el spiritu s(an)to viue y rreina Dios por $/[[$ to $]$ dos los siglos de los siglos. Amen.

Çhaymantam tahua çhunca punçhaumanta Virgen Ma-/ria mamançhicpa ñauquimpi llapa sancto Apostolcunap ricus-/can hanacpaçhaman viçhay ripurcan, çhaypim llapa atipac / Dios yayap pañañequempi tiay<c>um. Hanac paçhaman viçhay ripuptinmi Angelcuna açhca huaranca vraycumuspa tari-/pamurcan. Paycaman jesus yayançhic hanacpaçhaman/rircan, manam pusascaçhu viçharircan. çhay Angelcu-/nari çhunca huaranca huñu yallequeyocmi vraycumuspa / cusicurcancu.

Cay chica nanac Angelcunap catiscanmi jesus yayan-/çhic gloriaman ripurcan, Sepraphincunactapas yallis/pam pahuarcan, huayramantapas yallin pahuarispam / viçhay ripurcan: sanctocunap ñaupaquenta rispam ñanta / purircan, gloria puncuctapas quiçharircanmi, paypa / llamancuna yaucuncancupac. 
Muçhaycuscayqui apu yaya jesu xp(ist)o hanac gloriaman / cusicuy paçhaman ripucta: muçhaycuyquitacmi yaya / Diospa cayllampi cunan tiaycucta. Huçhasapa çhurijquic/ta, yaya ama concallahuayçhu, quespiçhequeymi yaya. Pas-/torniymi canqui: çhincac llamayquicta ama saquerihuanquiçhu, hamuy ñillahauytac (8) capac Dios yaya: Dios yaya-/manmi. llapa soncoyhuan I ñini, sapay çhurin jesu / xp(ist)o Diosman I ñicunitacmi, spiritu sancto viñay cac / Diosmampas i ñinitacmi./

Spiritu sancto Capac Diosmi, piçhca çhunca punçhau / yalliscanmanta Apostolcunaman vraycumurcan Pente-/costes punçhaupi: jesus yayançhic hanac paçhaman ripup- (9)/tin ñam, spiritu sanctocta suyacuncampac Apostol/

$<\mathrm{cu}->/ /$

(f300v)cuna ñatac jerusalenman cutimurcan Ayunacuspa [[rre $] /$ sacuspa Diosta muçhacuspa camam, virgen Maria sapay/mamançhichuan huaqilla carcan huaci vcupi.

Concayllamantam cana Spiritu sancto capac Dios / ninacallu hinalla hanacpaçhamanta çapaçapaman / vraycumurcan. çhay huacictari capac cayninhuan hun-/taycuçhircanmi Spiritu sancto Dios, animantapas coçhu-/çhipurcanmi, graçianhuampas huntaycuçhircanmi. Runa-/cunari Kac ñicta vyarispam, ymanançha ñispa tanta-/nacurcancu: Apostolcunactari llapantinpunim vyarir-/cancu Diospa simin rimacta.

Ierusalen llactapim; hinantin paçhapi cac ayllu/cuna Pascua punçhaupi tantanacurcan Diosta muçhan/capac. Apostolcuna capac Diospa siminta rimaptinmi may-/can ayllu caccunapas, llactampa siminpi cunascanta / vyarircancu. Spiritu sancto Diospa yaçhaçhiscanmi Apos/tolcuna paycunap simimpi suti rimarcancu. Apostolcunac/ ta predicacta vyarispa, ançha nanac runam Diosman,/ cutiricurcancu.

Spiritu Sancto huacçhacunap yayan, huacçha ru-/nayquiman hamullahuaytac, rumi soncoyta llampuya-/çhipuaytac, huçhaycunamanta huanaçhihuaytac Spiri-/tu sancto Diospa raurac ninan, caç * (10) amullahuaytac / cançhac ninayquicta tulluy vcuman, yaçhaçhillahuaytac / camta huaylluyta. Spiritu sancto Dios campa suyuyquiman / acllasca ayllu Cristianoiquicunaman paramullahuay, / capac graçiayquicta sullamuaytac.

Spiritu sancto Dios, canmi capac Dios yayahuan, ca-/pac Dios çhurihuan huc sapallan Dios canqui: Huçhay-/cucta pampaçhapuaycu huçhapampaçhac Dios yaya / spiritu sancto saycucta yanapac yanapahuaycu llassac / huçhacunahuan saycucta : Huçhaycunahuan, huçha ñanta //

(f301r) [[puri]]spa ançham saycuycu. Spiritu sancto causac Vnu pucyu / mapa soncoyta mayllaripullahuay: çhaquic animaytari çac-/çaçhipullahuaytac capac graçiayquihuan. Spiritu sancto/Dios animay çhacracta, huañuc mallquicta carparipulla/huay: cam Dios yaya parcopuaptijquica inquillcunallam / viñay çiçamunca.

Cay quellcascayta çhurijcuna huc ancha / collanan simihuan huntaçhipuscayquiçhic: / Capac jesu xp(ist)o yayançhicmi: paçha puçhucaptin / tucuy
(8) Metátesis de ñillahuaytac.
(9) Nota al margen: Act. 2.
(10) Mancha. 
runacunacta taripac hampunca, hua-/ñuccuna causarimpuncam: taripancam jesu-/xp(ist)o Dios yaya llapan runacunacta, llapa runa-/cunacta pataçhacmi vraycumunca, rurascan/çhic camam taripahuasun: Alli runactam vi-/ñaypac coçhuçhinca, mana allicunactam rupac / ninaman viñaypac carcunca -

Taripay punçhaupim çhurijcuna llapançhic cau-/sarimusun. cay quiquin vcunçhichuan, ñauinçhichuan / maquinçhichuan, tullunçhichuanmi, cay quiquin ayçhan/çhichuan, carançhichuanmi causarimusunçhic: Manam / ymallapas çhincapuasunçhu, manam huc çhucçhallapas / hucpa çhucçhanhuan rantinacuncachu, Diospa capac call/panraycumanta. Na tucuy hinantin runacuna Diospa si-/minta vyarispa causarimuptinmi cana, Dios yaya Angel/nincunacta caçhamunca, huc paçhallapi llapampas tanta-/ nacuncampac. Çhaypi paçha callariscanmanta puçhuca-/nancama: cac, llapa hinantin runacuna tiaptinmi, hanac/paçhapi Sancta Cruspa quepanta huaranca huñu Angel/cuna Sanctocunapas catimunca, maquimpi tupasca yspa-/rayoc cama, çhaycunap çhaupimpim jesu xp $($ ist $)$ o yaya capac / [[Dios]]pa çhurin, tucuy hinantimpa taripaquençhic ham-/

$<$ punca $>/ /$

(f301v)punca tucuy hinantinpa mançhanan pay quiquin [[hamuncam]]/ hanacpaçhacunapas payta ricuspaca mançharispa / cuyuncam.

Runacunari jesus yayançhictaricuspallam/ñauinta allpañecman cumuycuçhinca, huañuchinam / mançharispa vtirayanca, çhay paçham ñinca: Orcocuna / ñitihuayçhic, paçha millpuay, pacahuay, amatac Di[[o]]spa / piñacuyñinta ricusacçhu. Hinam jesu $\mathrm{xp}($ ist $) \mathrm{o}$ Dios yaya ha-/munca capac tianampi tiacuspa: Angelcunap, sancto/cunap çhaupiçhascan: çhaymi Angelcunacta camaçhir/cañispa: Alli runacta mana allicunamanta raquircuy / acllarcuy, ñiptillanmi. Alli runacunaca mana allicuna/manta pañañecman raquircucunca, mana allicunam / lloqueñecpi tiaycunca. Atha, yma llaquijtam çhay $\mathrm{ma}[[-]] /$ na allicuna llaquinca, viñaypac ñacaricuy allparicuy / ñecman visçhurcusca: Atha .yma putijmi sonconcu-/nacta aparinca, çhucucucuspallam quiruntapas cata-/ tataçhinca, pana Inca cacpas, pana Capac cacpas.

Çhaypim çurijcuna Diospa quellcan ricurinca, / tucuypa alli rurascan, mana alli rurascancunapas çhay-/pi ricurincatacmi: Diospa siminmantam pi maycan cac/llapas, causascantapas llapanmi yaçhanca, chayta ri-/cuspam hanac paçhapi viñaypac caucaypac (11) camantapas / mana ñispa vcupaçhapi viñaypac ñacaricuypac camanta/pas yaçhanca. Atha yma yuyaycuytam yuyaycunca ma-/na alli runacuna, huçhasapa. payñecman capac taripac / vyanta piñacucta ricuçhiptin, piñac ñauinhuan ca-/huaycuptin, may çhay ñauihuan ñispa rimaptin. Rij-/çhic, hatarcoyçhic aucaycuna vcupaçha ninaman. supay/ cunahuan çhaypi viñaypac ñacaricuc viñaypac alla/paricuc.

Diospa çhurin jesu xp(ist)o yayançhicri cuyapayacuc ña/uinta llampulla, munay, munaylla vyanta cuyascancu///

(f302r) [[naman cutirichi] ]spam, misqui [[misqui]]lla napaycunca / [[...]. Hacu ñocahuan yayaypa munascancuna, caçhic (12) , cay-/[[...]] hanacpaçha camcunapac
(11) = cauçaypac
(12) $=c a[$ nqui] $\operatorname{chic}(?)$. 
camariscay, camcunapac hua-/[[c]]ayçhapuscay ñispam ñinca. May yma cusicuyçha çhay / cusicuyman pactanman? Soncocunapas cusicuypa huntas-/canmi canca, llapanri ançha coçhucuspa, cusicuspam Dios-/man sonco canca, viñaypactac cusicunca, ñacaricuscanta / puçhucacta ricuspa. Mana ñam hanacpaçhapica yarecay / cançhu, manam oncoy cançhu, huacçha caypas, saycuypas, / huañuypas manam cançhu, manam puticuy, llaquicuy / yma yuyaypas cançhu, cayçhu, çhayçhu canca ñijpas cançhu, / manam çhaypica huçhapas huatecaypas, llaquicuypas, llullacuypas, tacuricuypas cançhu, hucta huçhalliçhij-/pas gloriapica manam cançhu.

Cay tucuyca puçhucanman, ñam paçha cutin, çhaypica / mana tucuc, mana pisipac cusicuyllañam, cuyacuyllañam, / Diosta munayllañam can, ñam mosoc paçha çhayamun, / çhay paçhaca viñaypac samacuspa cauçay paçham çhay / cusi llactapi, çhay ñauraycuna çiçac huayllapi, çhay / causac vnu quitipi, çhay cusicuy mayu Dios[[ñ]] ecmanta lloc-/sicpi, çhaytam viñaypac mana samaspa vpian, viñay/vpiaspapas ashuanracmi misquin, viñayllam Diospa / cusicuynincunacta vpian. Çhay sanctocunap vcuncunari / huamancunamantapas ashuan pahuacmi, intimantapas / ashuan sumacmi, huayramantapas ashuan puricmi canca / hanac paçhactapas Çumaspam yallinca, Animancunam / Dios yayançhichina canca Diospa huntaycuscan, Angelcu-/nahuan pactallam, cuscallam Diosnimpa, yayampa cuyas-/can çhurin camam canca.

Gloriapica llapampas cuyanacunca, huayllunacunca ca-/mam, çapamantari, huaquinninman cusicuyninta, sama-/cuyninta conacunca camam, llapanri hucllap cusicuynin-/mantapas cusicunca camam. çapamantari llapampa cusi-/cuscanmantam cussicunca. Tucuymantapas caru yallic-/ cusicuyninca Diosta ricuymi, paypa sumac cayninta, ymay-/mana - caquenta, cusicuynintahuan ricuspa cusicuymi. / Manam cayca huauque yuyayllahuampas yuyaypacçhu / [[y]]ariçhus rimaypac villacuypac canman. Manam pi may-/[[quempa]] rinrimpas vyarinçhu ñauimpas manam ricunçhu

$$
<\text { yuyay-> // }
$$

(f302v) yuyaynimpas manam yuyanchu, [[imay $]] /$ mana caquencunacta munaquen yupaychaquencuna/pac huacaychapuscanta

Chay ari çhurijcuna. cunanmantaca Diosnin-/çhicta tucuy soncoyquiçhichuan munayçhic, tucuy call-/payquiçhichuan. siruijçhic, pana huañuyhuampas/camaçhiscan siminta huacayçhayçhic. Cay villascay-/mi çhurijcuna feeninçhic, y ñincançhic caymi, cayta / pi cacpas mana i ñiptinca. manam viñaypac yaçha-/cupunmançhu Diosman quespinan. Cayta ruraspaca / ançha cusiocpunim canquiçhic Diospa cuyascan çhu-/ rinmi canquiçhic, huañuspapas çhay çhica allin, çhica / cusicuy viñay causaymanmi rinqiçhic, çhaytam cos-/cayquiçhic ñispam jesu xp(ist)o Dios yayançhic. siruiquen-/ cunaman nin.

Paymi Dios, yayahuan, Dios spiritu sanctohuan vi-/ñaypac causanca. Amen-

$$
* * *
$$

Todo lo diçho y contenido en estos ser-/mones, y traducçion de los sanctos Euange-/lios offreçe el Auctor a la correcçion / y çensura de la sancta Madre Yglesia / Catholica, Apostolica, Romana. - 
La traducçion de los sanctos Euangelios / segun la letra y el sentido ba rregulada en / todos los terminos con el idioma latino, / y correcta segun el pareçer de las personas

$<m a s>/ /$

(f303r) [[más hábiles que he podido]] hallar en la lengua, con los / [[cu]]ales se a tratado y comunicado y difficul-/tado muçhas veçes y la an aprobado saçer-/dotes clerigos y Religiosos los mejores / lenguas que e podido comunicar, A seglares / montañeses, e yndios muy ladinos a pare-/cido muy propia la traducçion, asi en Li-/ma Guamanga, Guanuco y Xauxa y en / otras partes. En todo e procurado siempre / seguir la letra y el sentido y quando no/ fuere possible seguir la letra, jamas abra/falta en seguir el sentido.

Y los sermones en la lengua general q(ue) con / muçho estudio y trauajo de dia y de noçhe por / espaçio de algunos años e escrito offresco con / muy grande voluntad a nuestros muy amados / compresbiteros y saçerdotes de $\mathrm{N}$ (uestro) señor / jesu xp(ist)o, confiando de su christiana pruden-/cia y charidad lo rrecebiran piadosamente / y escusaran los yerros que hallaren en este / camino nueuo que e auierto, por el qual nin/guno se que aya caminado antes de aora.

Al mejor pareçer, e juizio y sentido catho-/lico estoy muy sujeto para rreçeuir la / correcçion y enmienda de quien la sepa dar / y entienda: superandome ante todas cossas / con el alma y coraçon y captiuando el en-/tendimiento, los pensamientos, palabra //

(f303v) y escritos en latin, rromance [[castellano]] / y lengua queçhua y en todo quanto hablar[[e,]]/ escribiere, enseñare, o predicare a la / çensura y correcçion de N(uestra) sancta Madre / Yglesia Catholica Romana, Apostolica / Columna y Regla de verdad: Cuya fee / y Doctrina protesto guardar y confiesso / tener, sentir y creer y en ella viuir y morir / assi como eon toda la vniuersal yglesia/ catholica. y los sanctos Conçilios plenarios / y generales. Conuocados y confirmados por / el sanctissimo Pontifice Romano lo abra-/çan, sienten, tienen. y creen en vn solo / Dios verdadero Padre. Hijo. y Spiritu sancto / vna en essençia y Trino en personas. / Al qual sea honrra, Bendiçion, claridad / sabiduria, y hacimiento de graçias, honor vir/tud y fortaleza por todos los siglos de / los siglos: Amen. — Jesus M(ari)a

Acabose esta obra a los seis de Febrero del / año de 1649. dia de la Epiphania de $\mathrm{N}$ (uestro) s(eñor) jesu xp(ist)o / sea para gloria y honra de Dios todo poderoso / y de la Bien auenturada siempre virgen sancta / Maria nuestra señora Conçeuida sin macula / de pecado original. y de mi seraphico Padre / san Françisco, cuyo hijo humilde aunque //

(f304r)[[...]] estos sermones para el bien/ [[y]] proueçho de las almas de los pobres yn-/dios del Piru: y pide el Auctor por amor / de Dios le perdonen las faltas, y le enco-/mienden a Dios y le rreçen la oraçion de / la sabana sancta con q(ue) se saca cada / ves q $($ ue $)$ le dixere vna Anima del Purgato-/rio.

La sanctidad de Clemente VIII. conçedio a petiçion / de la sereniss ( $\mathrm{im}$ )a Infanta Doña Catalina, que todas las / veçes que se rreçare esta Oraçion en rreuerençia de / la sancta Sabana, en que fue enbuelto el cuerpo / sanctiss (im)o de N(uestro) s(eñor) jesu $\mathrm{xp}($ ist $)$ o se saque vna Anima de / Purgatorio. - 
Apu yaya Iesu christo, canmi cay paçhapi / ñocaycupac ñacaricuscayquip vnançhanta / yurac paçhapi saquercanqui, çhayhuanmi ari / sanctissimo ayayqui pintusca carcan Crusman-/ta joseph vraycuçhimuptin: Cuyaycuaycu cu-/yapayacuc yaya huañuscayquiraycu, pampas-/ca cascayquiraycupas gloriayqui cussi paçha-/man pusasca cancaycupac. Cam Diosmi capac / causanqui. Dios yayahuan, Dios spiritu sancto-/huampas huclla caynijquipi; mana puçhucac / viñay Causaynijquipi. Amen [[firma $]$ Diego de Molina / Peña 


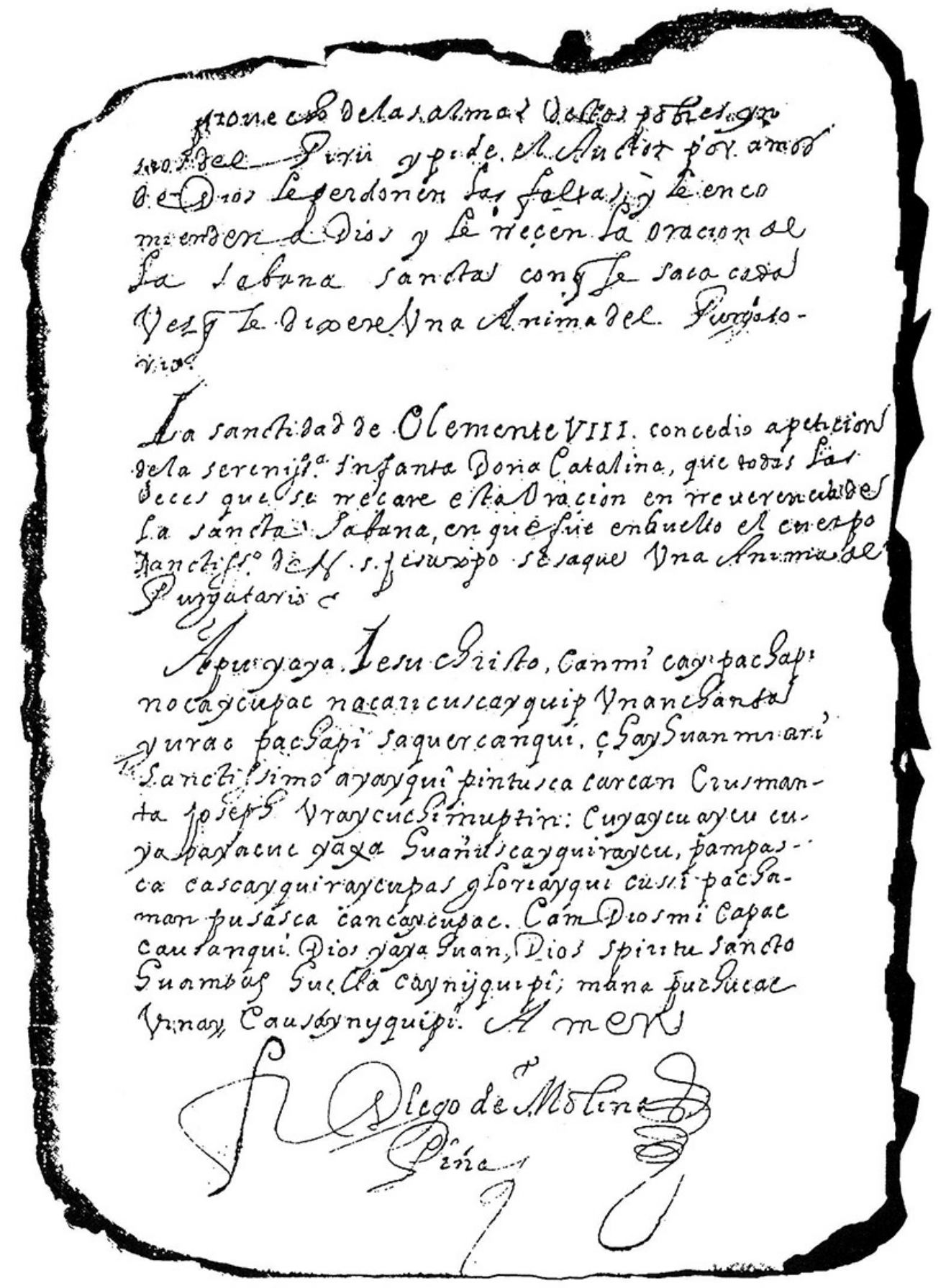




\section{VERSIÓN NORMALIZADA DE LOS TEXTOS QUECHUAS}

Chaymantam tawa chunka punchawmanta Virgen María mamanchikpa ñawkinpi llapa Santo Apóstolkunap rikuŝqan hanaqpachaman wichay ripurqan. Chaypim llapa atipaq Dios yayap pañañiqinpi tiyaykun. Hanaqpachaman wichay ripuptinmi Ángelkuna achka waranqa uraykumuŝpa taripamurqan. Paykaman Jesús yayanchik hanaqpachaman rirqan. Manam puŝâ̂qachu, wicharirqan. Chay Ángelkunari chunka waranqa huñu yalliqiyuqmi uraykumuŝpa kuŝikurqanku.

Kay chika nanaq Ángelkunap qatiŝqanmi Jesus yayanchik gloriaman ripurqan. Serafínkunaktapâ̂ yalliŝpam pawarqan. Wayramantapaŝ yallin pawariŝpam wichay ripurqan. Santokunap ñawpaqinta riŝpam ñanta purirqan. Gloria punkuktapâ̂ kicharirqanmi paypa llamankuna yawkunqankupaq.

Muchaykuŝqayki, apu yaya Jesucristo, hanaq gloriaman kuŝikuy pachaman ripuqta. Muchakuykitaqmi, yaya, Diospa qayllanpi kunan tiyaykuqta. Huchasapa churiykikta, yaya, ama qunqallawaychu. Qiŝspichiqiymi, yaya, pastorniymi kanki. Chinkaq llamaykikta ama saqiriwankichu. Hamuy, ñillawaytaq, qapaq Dios, yaya. Dios yayamanmi llapa ŝunquywan iñini. Sapay churin, Jesucristo, Diosman iñikunitaqmi. Espíritu Santo wiñay kaq Diosmanpaŝ iñinitaqmi.

Espíritu Santo qapaq Diosmi pichqa chunka punchaw yalliŝnanmanta Apóstolkunaman uraykumurqan. Pentecostés punchawpi Jesús yayanchik hanaq pachaman ripuptin ñam Espíritu Santokta ŝuyakunqanpaq Apóstolkuna ñataq Jerusalénman kutimurqan. Ayunakuŝpa rezakuŝpa Diosta muchakuŝpa kamam virgen María sapay mamanchikwan wakilla karqan wasi ukupi.

Qunqayllamantam qana Espíritu Santo qapaq Dios ninaqalluhinalla hanaqpachamanta sapa sapaman uraykumurqan. Chay wasiktari qapaq kayninwan huntaykuchirqanmi. Espíritu Santo Dios ánimantapas quchuchipurqanmi, gracianwanpas huntaykuchirqanmi. Runakunari qaq ñiqta uyariŝpam, "imananchá" ñiŝpa tantanakurqanku. Apóstolkunari llapantinpunimuyarirqanku Diospa ŝimin rimaqta.

Jerusalén llaqtapim hinantin pachapi kaq ayllukuna Pascua punchawpi tantanakurqan Diosta muchanqa[n]paq. Apóstolkuna qapaq Diospa ŝiminta rimaptinmi, mayqan ayllu kaqkunapaŝ, llaqtanpa ŝiminpi kunaŝqanta uyarirqanku. Espíritu Santo Diospa yachachiŝanmi Apóstolkuna paykunap ŝiminpi ŝuti rimarqanku. Apóstolkuna predicaqta uyariŝpa ancha nanaq runam Diosman kutikurqanku. 


\section{Traducción CaStellana de la Versión QUEChUA NORMALIZADA}

Luego, después de cuarenta días, ante los ojos de Nuestra Madre y a la vista de todos los santos Apóstoles, [Jesús] subió al Cielo. Allí se sentó a la derecha de Dios padre, el todopoderoso. Mientras subía al Cielo, millares de Ángeles bajaron a su encuentro. Por su propia voluntad, nuestro padre (1), Jesús, se fue al Cielo. No fue conducido, subió [sin ninguna ayuda]. Y esos Ángeles, más de diez mil todos juntos, bajaron y se alegraron.

Seguido por esta enorme compañía de Ángeles, nuestro Padre Jesús subió a la Gloria. Voló más rápido que los Serafines. Más rápido que el viento subió volando. Siguió su camino por delante de los Santos y abrió la puerta de la Gloria para que su grey pudiese entrar.

Te adoro, o Padre Señor Jesucristo, mientras subes hacia la Gloria, al país de la Beatitud. Y te adoro, o Padre, ahora que estás sentado al lado de Dios. No te olvides, o Padre, de mí, tu hijo lleno de pecados. Padre, tú eres mi salvador, mi pastor. No me abandones a mí, la oveja perdida. Ven, dímelo, o Dios poderoso, Padre. Creo de todo mi corazón en Dios Padre. También creo en su único hijo, Jesucristo, Dios. De igual manera creo en el Espíritu Santo, Dios Eterno.

Después de haberse cumplido cincuenta días, bajó el Espíritu Santo, Dios poderoso, donde los Apóstoles. En el día del Pentecostés, cuando Nuestro Padre Jesucristo ya había subido al Cielo, los Apóstoles volvieron a Jerusalén para esperar al Espíritu Santo. Estaban todos juntos en una casa con nuestra única Madre, la Virgen María, ayunando, rezando y adorando a Dios.

De repente, el Espíritu Santo, Poderoso Dios, semejante a una lengua de fuego, bajó del Cielo a cada uno de ellos y llenó esa casa con su majestad. Dios, el Espíritu Santo, regocijó sus ánimas y las llenó con su gracia. Y los hombres, al oír el sonido de un trueno, se preguntaron qué podía ser y se reunieron. Y los apóstoles todos también oyeron la voz de Dios que les hablaba.

En la ciudad de Jerusalén, todas las tribus que había en el país, se juntaron para adorar a Dios en el día de Pascua. Cuando los Apóstoles comunicaron la palabra de Dios poderoso, todas las tribus escucharon su predicación en el idioma de su propio país. Los apóstoles, instruidos por Dios, el Espíritu Santo, revelaron la verdad en el idioma de cada una de ellas. Al escuchar la prédica de los Apóstoles, muchísima gente se convirtió a [la fe de] Dios (2).

(1) Molina emplea la palabra yaya indiscriminadamente para referirse a cualquiera de las tres personas. Sin embargo, también distingue a 'Dios Padre' de Dios Hijo y Dios Espíritu Santo. Es posible que su empleo de 'padre', al referirse al Hijo y al Espíritu Santo, corresponda al matiz 'señor' que también expresa el lexema yaya. En esta traducción, mantenemos el término 'padre' como glosa de yaya en todos los contextos.

(2) Hay varias interpretaciones posibles del texto quechua. 'Muchísima gente volvió a Dios', es decir que los que habían cesado de creer, fueron conquistados por el impacto de la prédica o que los judíos comprendieron, gracias a la elocuencia inspirada por el Espíritu Santo, que la religión de Dios, es decir: Jesucristo, era la verdadera. 
Espíritu Santo wakchakunap yayan, wakcha runaykiman hamullawaytaq; rumiŝunquyta llampuyachipuwaytaq; huchaykunamanta wanachiwaytaq; Espíritu Santo Diospa rawraq ninan kachamullawaytaq; kanchaq ninaykikta tulluy ukuman yachachillawaytaq qamta; waylluyta Espíritu Santo Dios qampa ŝuyukiman akllaŝqa ayllu Cristianoykikunaman paramullaway, qapaq graciaykikta ŝllamuwaytaq.

Espíritu Santo Dios, qa[m]mi qapaq Dios yayawan, qapaq Dios churiwan huk sapallan Dios kanki. Huchaykuqta pampachapuwayku. Huchapampachaq Dios yaya, Espíritu Santo ŝykuqta yanapaq yanapawayku llaŝaq huchakunawan saykuqta. Huchaykunawan, hucha ñanta [puri]spa ancham ŝaykuyku. Espíritu Santo kawsaq unu pukyu mapa ŝunquyta mayllaripullaway. Chakiq ánimaytari saksachipullawaytaq. Qapaq graciaykiwan Espíritu Santo Dios, ánimay-chakrakta wañuq mallkikta qarparipullaway. Qam, Dios yaya, parqupuwaptiykiqa inkillkunallam wiñay sisamunqa.

Kay qillqaŝqayta, churiykuna, huk ancha qullanan ŝimiwan huntachipuŝsqaykichik. Qapaq Jesús Cristo yayanchikmi pacha puchukaptin tukuy runakunakta taripaq hampunqa. Wañuqkuna kawsarimpunqam. Taripanqam Jesucristo, Dios yaya, llapan runakunakta. Llapa runakunakta patachaqmi uraykumunqa. Ruraŝqanchik kamam taripawaŝun. Alli runaktam wiñaypaq quchuchinqa. Mana allikunaktam rupaq ninaman wiñaypaq qarqunqa.

Taripay punchawpim, churiykuna, llapanchik kawsarimuŝun. Kay kikin ukunchikwan, ñawinchikwan, makinchikwan, tullunchikwanmi, kay kikin aychanchikwan, qaranchikwanmi kawsarimuŝunchik. Manam imallapaŝ chinkapuwaŝunchu. Manam huk chukchallapaŝ hukpa chukchanwan rantinakunqachu Diospa qapaq kallpanraykumanta. Ña tukuy hinantin runakuna Diospa siiminta uyariŝpa kawsarimuptinmi qana Dios yaya Ángelninkunakta kachamunqa huk pachallapi llapanpa $\hat{s}$ tantanakunqanpaq. Chaypi pacha qallariŝqanmanta puchukanankama kaq llapa hinantin runakuna tiyaptinmi hanaqpachapi Santa Cruzpa qipanta waranqa huñu Ángelkuna Santokunapâ̂ qatimunqa makinpi tupaŝqa isparayuqkama. Chaykunap chawpinpim Jesucristo, yaya qapaq Diospa churin, tukuy hinantinpa taripaqinchik hampunqa. Tukuy hinantinpa manchanan pay kikin hamunqam, hanaqpachakunapaŝ payta rikuŝpaqa manchariŝpa kuyunqam.

Runakunari Jesús yayanchikta rikuŝpallamñawinta allpañiqman kumuykuchinqa. Wañuqhinam manchariŝpa utirayanqa. Chay pacham ñinqa: "Urqukuna ñitiwaychik, pacha millpuway, pakaway, amataq Diospa piñakuyninta rikuŝaqchu". Hinam Jesucristo Dios yaya hamunqa qapaq tiyananpi tiyakuŝspa Ángelkunap Santokunap chawpichaŝqan. Chaymi Ángelkunakta kamachinqa ñiŝpa: "Alli runakta mana allikunamanta rakirquy, akllarquy". Ñiptillanmi: “Alli runakunaqa mana allikunamanta pañañiqman rakirqukunqa, mana allikunam lluqiñiqpi tiyaykunqa”. Atha, ima llakiytam chay mana allikuna llakinqa wiñaypaq ñakarikuy allparikuyñiqman wischurquŝaa. Atha, ima putiymi ŝnqunkunakta aparinqa, chukukukuŝspallam kiruntapaŝ katatatachinqa, pana inka kaqpaŝ, pana qapaq kaqpaŝ. 
Espíritu Santo, padre de los pobres, ven hacia mí, hombre menesteroso; ablanda mi corazón de piedra; hazme enmendar de mis pecados; Espíritu Santo, fuego ardiente de Dios, envíame tu fuego iluminante para que penetre mis huesos; enséñame lo que eres; Espíritu Santo Dios, haz llover amor sobre tus partidarios, el pueblo escogido de los cristianos tuyos, haz caer sobre nosotros el rocío de tu gracia poderosa.

Dios Espíritu Santo, tú que, junto con el poderoso Dios padre y el poderoso Dios hijo, eres un solo Dios, perdónanos nuestros pecados. O Dios Padre, tú que perdonas los pecados, Espíritu Santo, que ayudas a los cansados, ayúdanos a nosotros, cansados por el peso de nuestros pecados. Mucho nos hemos cansado al caminar por el camino del pecado, cargando nuestros pecados. Espíritu Santo, manantial de agua viva, limpia mi corazón sucio. Harta mi ánima sedienta. Riega con tu poderosa gracia, o Espíritu Santo Dios, las plantas de la chacra de mi ánima que mueren. Si tú, Dios Padre, me la riegas, sólo brotarán flores, siempre.

Terminaré lo que he escrito (3) aquí para ustedes con unas palabras de suma importancia. Nuestro poderoso padre Jesucristo, al acabarse el mundo, volverá para juzgar toda la gente. Los muertos resucitarán. Jesucristo, Dios Padre, juzgará a toda la gente. Bajará para evaluar a toda la gente. Juzgará todos nuestros actos. Al hombre bueno le ofecerá el gozo eterno. A los malos los expulsará hacia el fuego ardiente donde padecerán para siempre.

En el día del Juicio, hijos míos, nosotros todos resucitaremos. Con este mismo cuerpo, estos mismos ojos, estas mismas manos, estos mismos huesos, esta misma carne y esta misma piel, resucitaremos. Ninguna parte de nosotros faltará. Gracias a la fuerza poderosa de Dios, ni un solo de nuestros cabellos se cambiará con los cabellos de otro. Cuando todos los seres humanos, al escuchar las palabras de Dios, se hayan resucitado, entonces Dios Padre, enviará a sus ángeles para que todos se reúnen en un solo lugar. Cuando estén allí todas las personas que hayan existido desde el inicio hasta el fin del tiempo, en el cielo aparecerán miles de millares de ángeles y santos siguiendo a la Santa Cruz, cada uno con una espada afilada en su mano. En medio de ellos, Jesucristo, el hijo del poderoso Dios padre, volverá para juzgarnos a todos. Inspirando temor y veneración a todos, él mismo vendrá y, al verlo, hasta los cielos temblarán de miedo.

Y los hombres, sólo con ver a nuestro padre Jesús, bajarán sus ojos al suelo. Como si fueran moribundos, se desmayarán de miedo. Entonces dirán: 'Cerros, aplástenme, tierra, trágame, escóndeme para que no vea la ira de Dios'. Así vendrá Jesucristo Dios y se sentará sobre su trono en medio de los ángeles y los santos. Mandará a los ángeles que escogen y separen a los buenos de los malos. Y cuando diga: 'Las personas buenas serán apartadas a mi derecha, los malos estarán a mi izquierda', ¡ay, qué sufrimiento padecerán los malos, arrojados al dolor, a la pena eterna! ¡ay, qué tristeza agobiará sus corazones! temblarán y crujirán sus dientes aunque sean incas, aunque sean poderosos señores.

(3) Lit. 'Este mi escrito...'. El texto castellano original se refiere a 'este sermón'. 
Chaypim, churiykuna, Diospa qillqan rikurinqa. Tukuypa alli ruraŝqan, mana alli ruraŝqankunapaŝ chaypi rikurinqataqmi. Diospa ŝiminmantam pi mayqan kaqllapaŝ kawsaŝqantapaŝ llapanmi yachanqa. Chayta rikuŝpam hanaqpachapi wiñaypaq kawsaypaq kamantapaŝ mana ñiŝpa ukupachapi wiñaypaq ñakarikuypaq kamantapaŝ yachanqa. Atha, ima yuyaykuytam yuyaykunqa mana alli runakuna, huchasapa, payñiqman qapaq taripaq uyanta piñakuqta rikuchiptin, piñaq ñawinwan qawaykuptin, may chay ñawiwan ñiŝpa rimaptin: "Riychik, hatarquychik, awqaykuna, ukupacha ninaman supaykunawan chaypi wiñaypaq ñakarikuq wiñaypaq allparikuq.

Diospa churin Jesucristo yayanchikri kuyapayakuq ñawinta llampulla, munay munaylla uyanta kuyaŝqanku[naman kutirichi]ŝspam, miŝki [miŝki]lla napaykunqa [ñispa]: 'Haku ñuqawan, yayaypa munaŝqankunakan[ki]chik. 'Kay[mi kay] hanaqpacha qamkunapaq kamariŝ̀ay, qamkunapaq waqaychapuŝsqay' ñiŝpam ñinqa. May ima kuŝikuycha chay kuŝikuyman paqtanman? Eunqukunapaŝ kuŝikuypa huntaŝqanmi kanqa. Llapanri ancha quchukuŝpa, kuŝikuŝpam Diosman ŝunqu kanqa. Wiñaypaqtaq kuŝikunqa, ñakarikuŝanta puchukaqta rikuŝpa. Mana ñam hanaqpachapiqa yariqay kanchu. Manam unquy kanchu. Wakcha kaypaŝ, ŝaykuypaŝ, wañuypaŝ manam kanchu. Manam putikuy, llakikuy, ima yuyaypaŝ kanchu. [Manam] "kaychu, chaychu kanqa" ñiypaŝ kanchu. Manam chaypiqa huchapaŝ watiqaypaŝ, llakikuypaŝ, llullakuypaŝ, takurikuypaŝ kanchu. Hukta huchallichiypaŝ gloriapiqa manam kanchu.

Kay tukuyqa puchukanman. Ñam pacha kutin. Chaypiqa mana tukuq, mana pisiipaq kuŝikuyllañam, kuyakuyllañam, Diosta munayllañam kan. Ñam muŝuq pacha chayamun. Chay pachaqa wiñaypaq samakuŝpa kawsay pacham. Chay kuŝsi llaqtapi, chay ñawraykuna sisaq wayllapi, chay kawsaq unu kitipi, chay kuŝikuy mayu Diosñiqmanta lluqsîiqpi, chaytam wiñaypaq mana samaŝpa upyan. Wiñay upyaŝpapas aswanraqmi miŝkin. Wiñayllam Diospa kuŝikuyninkunakta upyan. Chay Santokunap ukunkunari wamankunamantapaŝ aŝwan pawaqmi, intimantapaŝ aŝwan sumaqmi, wayramantapaŝ aŝwan puriqmi kanqa, hanaqpachaktapaŝ [s]umaŝpam yallinqa. Animankunam Dios yayanchikhina kanqa. Diospa huntaykuŝaan, Ángelkunawan paqtallam, kuskallam Diosninpa, yayanpa kuyaŝqan churin kamam kanqa.

Gloriapiqa llapanpaŝ kuyanakunqa, wayllunakunqa kamam. Sapamantari wakinninman kuŝikuyninta samakuyninta qunakunqa kamam. Llapanri hukllap kuŝikuyninmantapaŝ kuŝikunqa kamam. Sapamantari llapanpa kuŝikuŝsanmanta kuŝikunqa. Tukuymantapaŝ karu yalliq kuŝikuyninqa Diosta rikuymi. Paypa sumaq kaynintaimaymana kaqinta kuŝsikuynintawan rikuŝpa kuŝikuymi. Manam kayqa, wawqi, yuyayllawanpas yuyaypaqchu, yarichuŝ rimaypaq willakuypaq kanman. Manam pi may[qinp]a rinrinpaŝ uyarinchu, ñawinpaŝ manam rikunchu, yuyayninpaŝ manam yuyanchu, [imay] mana kaqinkunakta munaqin yupaychaqinkunapaq waqaychapuŝqanta. 
Entonces, hijos míos, habrán de aparecer los [libros que contienen lo] escrito por Dios. Lo bueno hecho por cada uno y lo malo también serán revelados. Por la boca de Dios todos sabrán lo que cada uno haya sido y hecho. Al ver [lo que Él ha escrito], sabrán quiénes han merecido la vida eterna en el cielo o, al contrario, quiénes han merecido el castigo eterno en el infierno. ¡Ay, qué sentimientos habrán de probar las personas malas, llenas de pecados, cuando el Poderoso Juez dirigirá hacia ellos su rostro airado y los mirará con ojos llenos de rabia y, al juzgarlos (4), les dirá: 'Váyanse, aléjense, mis enemigos, a los fuegos del infierno para sufrir y padecer allá junto con los diablos para siempre!'

Y el hijo de Dios, nuestro padre Jesucristo, volviendo sus ojos misericordiosos y su rostro lleno de ternura y de amor hacia sus queridos, los saludará con palabras muy dulces: ‘ $¡ V e n g a n$ conmigo! Ustedes son los queridos de mi padre!' 'He aquí el cielo que he aparejado y guardado para ustedes’, les dirá. ¿Cuál felicidad podrá igualarse a ésta? Sus corazones estarán llenos de felicidad. Todos, llenos de gozo y de felicidad, dirigirán sus pensamientos a Dios. Su felicidad será eterna al ver sus sufrimientos acabarse. Ya no habrá hambre en el Cielo. No habrá enfermedad. Tampoco habrá pobreza, cansancio ni muerte. No habrá sufrimiento, lamentación ni preocupación de cualquier naturaleza. Tampoco habrá indecisión. Allá no hay pecado, tentación, lamentación, engaño, ni confusión. No existe en la Gloria la posibilidad de inducir otro en el pecado.

Todo esto se acabaría. Ya es el fin del tiempo (5). Aquí ya no hay más que felicidad y amor que nunca acaban, que nunca menguan, no hay más que amar a Dios. Ya ha llegado el tiempo nuevo. Ese tiempo de eterno descanso es el tiempo de la vida. En este país de felicidad, en esta pradera donde crecen todas las variedades de flores, en este territorio de agua viva, para siempre, sin pausa, beben [el agua que proviene de] este río de felicidad que tiene su fuente en Dios mismo. Aunque se la bebe eternamente, su sabor siempre es agradable. Eternamente beben las delicias que ofrece Dios. Los cuerpos de los santos vuelan más [veloces] que los halcones y más bellos que el sol, se desplazarán más que el viento y en su belleza aventajarán el Cielo. Sus almas serán semejantes a Dios Padre. Llenos de [la esencia de] Dios, serán iguales, idénticas a los ángeles, y todas serán hijos queridos del padre de Dios.

En la Gloria todos se amarán con compasión, con ternura. Cada uno cuidará de la felicidad y el sosiego de los demás. Todos serán felices contemplando la felicidad de los otros. Cada uno se regocijará al ver a los demás felices. De lejos la causa principal de su felicidad será la visión de Dios. El hecho de contemplar su belleza y todo su ser y la felicidad [que emana de] Él es en sí mismo felicidad. Sería imposible imaginarla, hermanos, ni aún menos describirla. No ha escuchado oído alguno ni visto ojo alguno ni concebido mente alguna la infinidad de cosas [que Dios] ha destinado a los que lo amen y le obedezcan.

(4) may chay ñawiwan ñiŝpa no es fácilmente comprensible. Es posible que, en este contexto, ñawi pueda interpretarse como "el fiel del peso" (Anón, 1586) y representar una balanza, como en las imágenes coloniales que evocan el Juicio Universal.

(5) El término pacha se refiere tanto al tiempo como al espacio. El fin del tiempo es el fin del mundo. El original castellano dice: "todo esto se acaba, pasó el tiempo y sus mudanzas...". 
Chay ari, churiykuna, kunanmantaqa Diosninchikta tukuy ŝunquykichikwan munaychik, tukuy kallpaykichikwan serviychik pana wañuywanpaŝ kamachiŝqan siiminta waqaychaychik. Kay willaŝaaymi, churiykuna, feeninchik; yñinqanchik kaymi. Kayta pi kaqpaŝ mana iñiptinqa manam wiñaypaq yachakupunmanchu Diosman qiŝspinan. Kayta ruraŝpaqa ancha kuŝiyuqpunim kankichik. Diospa kuyaŝqan churinmi kankichik. Wañuŝpapas chay chika allin, chika kuŝikuy wiñay kawsaymanmi rinkichik. "Chaytam quŝ̀aykichik" ñiŝpam Jesucristo Dios yayanchik serviqinkunaman [ñ]

Paymi Dios yayawan, Dios Espíritu Santowan wiñaypaq kawsanqa. Amen.

$* * *$

Apu yaya, Jesucristo, qa[m]mi kay pachapi ñuqaykupaq ñakarikuŝaaykip unanchanta yuraq pachapi saqirqanki. Chaywanmi ari santísimo ayayki pintuŝa karqan Cruzmanta Joseph uraykuchimuptin. Kuyaykuwayku, kuyapayakuq yaya, wañuŝqaykirayku, pampaŝqa kaŝqaykiraykupaŝ gloriayki kuŝi pachaman puŝâ̂qa kanqaykupaq. Qam Diosmi qapaq kawsanki Dios yayawan, Dios Espíritu Santowanpaŝ huklla kayniykipi, mana puchukaq, wiñay kawsayniykipi. Amen -//

[firma] Diego de Molina / Peña 
Entonces, hijos míos, a partir de hoy amen a nuestro Dios con todo su corazón, sírvanlo con toda su fuerza y guarden sus mandamientos aunque sea a costa de su vida. Estas cosas que les he contado, hijos míos, constituyen nuestra Fe; esto es lo que tenemos que creer. Quien no crea esto nunca beneficiará de la salvación de Dios. Si lo hacen, gozarán ustedes de mucha felicidad. Ustedes serán los hijos amados de Dios. Y, cuando mueran, irán a esta vida eterna tan buena y tan feliz. "Eso es lo que les daré", dice Jesucristo nuestro padre a los que le sirven.

Junto con Dios Padre y Dios Espíritu Santo, Él vivirá para siempre. Amen.

$* * *$

La oración de la sábana santa con que se saca cada vez que le dijere una ánima del Purgatorio.

Señor Padre Jesucristo, tú dejaste en este paño blanco la señal de lo que sufriste en este mundo para nosotros. Con éste tu santísimo cadáver fue envuelto cuando José lo bajó de la Cruz. Apiádate de nosotros, o Padre misericordioso, que, por tu muerte y tu sepultura, permitiste que fuéramos conducidos al bienaventurado país de tu Gloria. Tú eres Dios y vives poderoso, junto con Dios Padre y Dios Espíritu Santo con los que formas un solo ser, tu vida infinita, eterna. Amen - //

[firma] Diego de Molina / Peña

\section{Referencias citadas}

EGUIGUREN, L., A., 1949 - Diccionario Histórico-Cronológico de la Real y Pontificia Universidad de San Marcos y sus colegios, T. II; Lima.

ROMERO, C. A., 1928 - Un libro interesante. Revista Histórica, IX(1): 51-87; Lima. 